\title{
The Effect of Visual Feedback of Head Angles With Using a Mobile Posture-Aware System on Craniocervical Angle and Neck and Shoulder Muscles Fatigue During Watching the Smartphone
}

\author{
Su Jeong Kim'1, So Yeon Jeong', Tae Lim Yoon ${ }^{3}$ \\ 'Department of Physical Therapy, Ppuri Medical Center, Cheongju; ${ }^{2}$ Department of Physical Therapy, National Transportation Rehabilitation Hospital, \\ Yangpyeong; ${ }^{3}$ Department of Physical Therapy, College of Health and Medical Science, Cheongju University, Cheongju, Korea
}

Purpose: To find the effect of visual feedback of head angle with using a mobile posture-aware system on craniocervical angle and neck and shoulder muscles fatigue for preventing or decreasing the forward head posture.

Methods: Twenty-four healthy young adults in Chungbuk to participate in this study. The subjects started to watch a movie clip for 10 minutes with visual feedback in $0^{\circ}, 30^{\circ}$, and $60^{\circ}$ of head angles. During the task, surface electromyography (EMG) was used to collect data from the upper trapezius (UT), sternocleidomasetoid muscle (SCM), cervical erecter spinae (CES) during watching the smartphone. Craniocervical angles were measured using a sagittal-view photograph of the subject in a sitting posture. A one-way repeated analysis of variance with a significant level of 0.05 used for statistical analysis.

Results: Craniocervical angle with $0^{\circ}$ visual feedback was significantly greater than $30^{\circ}$ and $60^{\circ}$. Craniocervical angle with $30^{\circ}$ visual feedback was significantly greater than $60^{\circ}$. In addition, MDF of UT muscles in $0^{\circ}$ and $30^{\circ}$ of visual feedback was significantly greater than $60^{\circ}$.

Conclusion: We concluded that $0^{\circ}$ visual feedback of head angle with using a mobile posture-aware system would be beneficial to prevent or decrease forward head posture during watching a smartphone. We also could recommend using of $30^{\circ}$ visual feedback in case of caring UT muscle fatigue primarily.

Keywords: Electromyography, Forward head posture, Head up application, Muscle fatigue

\section{INTRODUCTION}

A forward head posture (FHP) is becoming more common with the increasing uses of smartphones. Recently, the smartphone has become a major item to enjoy a variety of media contents and applications for most people. ${ }^{1}$ In Korea, over 24 million people possessed a smartphone in 2013, and usually use an average of 4.1 hours a day on their smartphone, while heavy smartphone users spend 5.4 hours a day. ${ }^{2}$ However, many users may use their smartphones with their neck downwards to stare at the smartphone and sustain poor posture for a long periods of time. In detail, continuing a poor pos-

Received Mar 6, 2018 Revised Apr 17, 2018

Accepted Apr 26, 2018

Corresponding author Tae Lim Yoon

E-mail free0829@gmail.com ture in using a smartphone can be a source of various musculoskeletal disorders like neck muscle pain and FHP syndrome. ${ }^{3}$

FHP reduce cervical lordosis of the lower cervical vertebrae and usually related with restriction of the upper trapezius (UT) and cervical erector spinae (CES) as well as shortening of the sternocleidomastoid (SCM). ${ }^{2,4}$ Previous studies reported that the craniocervical angle (between a line through the spinous process of $\mathrm{C} 7$ and the tragus of the ear and a horizontal line through C7) is around 48.9853.68 anteriorly. ${ }^{5-7}$ Any decrease in craniocervical angle would indicate increasing of FHP.

To tackle this problem, several studies reported that various in-
Copylight ( 92018 The Korea Society of Physical Therapy

This is an Open Access article distribute under the terms of the Creative Commons Attribution Non-commercial License (Http:// creativecommons.org/license/by-nc/4.O.) which permits unrestricted non-commercial use, distribution, and reproduction in any medium, provided the original work is properly cited. 
terventions such as progressive exercise program, applied a series of manipulative procedures with an anterior head weight device, using of taping tension, sling correction exercise would decreased the FHP ${ }^{8-11}$ However, prevention method of FHP when using a smartphone was not sufficiently studied.

Recently, a new mobile posture monitoring system that could detect the physical behavior of smartphone users had introduced. The system periodically approximates the tilt angle of the user's neck during watching the screen with holding the smartphone in their hand. With the proposed system, users can be gain feedback of their neck angle by various methods such as vibration, text message, or sound alarm. ${ }^{3}$ Therefore, smartphone users could control their head angle with feedback from mobile posture monitoring system. Consequently, we fascinated to find the effect of visual feedback of head angle with using a mobile posture-aware system on craniocervical angle and neck and shoulder muscles fatigue.

The purpose of this study is investigation effect of visual feedback of head angles $\left(0^{\circ}, 30^{\circ}\right.$, and $\left.60^{\circ}\right)$ with using a mobile posture-aware system on craniocervical angle and neck and shoulder muscles (UT, SCM, and CES) fatigue during 10 minutes watching the smartphone screen for preventing or decreasing the forward head posture.

\section{METHODS}

G-power software was executed for power analyses. A necessary sample size of eight was calculated to achieve a power of 0.80 and an effect size of 0.52 (calculated with a partial $\eta^{2}$ of 0.209 from a pilot study with four subjects) with a level of 0.05 .

The subjects were 24 healthy young adults in Choongbuk. The descriptive characteristics of the participants are summarized in Table 1. The inclusion criteria were using a smartphone more than 3hours per day. And, subjects were excluded if they had (1) a history

Table 1. The descriptive characteristics of the participants

\begin{tabular}{lrcr}
\hline Variables & Male $(n=10)$ & Female $(n=14)$ & Total $(n=24)$ \\
\hline Age (year) & $19.82 \pm 0.92$ & $19.33 \pm 1.11$ & $19.53 \pm 1.02$ \\
Height $(\mathrm{cm})$ & $174.82 \pm 5.42$ & $158.54 \pm 5.01$ & $165.32 \pm 9.63$ \\
Weight $(\mathrm{kg})$ & $64.32 \pm 6.37$ & $54.13 \pm 5.13$ & $58.38 \pm 7.55$ \\
BMI $\left(\mathrm{kg} / \mathrm{m}^{2}\right)$ & $21.12 \pm 1.92$ & $21.51 \pm 2.12$ & $21.35 \pm 2.03$ \\
\hline
\end{tabular}

Values are presented as mean \pm SD.

BMl: body mass index. of severe surgical procedures and trauma such as spinal or lower limb fractures, neuromuscular disorder, whiplash injury, diabetic neuropathy, vestibular disease, dizziness, (2) abnormal findings confirmed through physical and neurologic examinations, and (3) congenital abnormalities in either the cervical or the lumbar spine. ${ }^{2,12}$ All subjects volunteered to participate in this study and signed an informed consent form.

\section{Equipment}

\section{1) Electromyography}

Noraxon TeleMyo-DTS and Noraxon MyoResearch 1.06 XP software (Noraxon, Inc., Scottsdale, AZ, USA) was used to investigate muscle fatigue in the right UT, SCM, and CES. Data for surface electromyography were amplified, bandwidth of 10-450 Hz, and notch filtered $(60 \mathrm{~Hz})$ before recorded with a sampling frequency of 1,000 Hz. The neck and shoulder area of the subjects were cleaned using alcohol swap and the hair was shaved. Then, the disposable $\mathrm{Ag} / \mathrm{AgCl}$ surface electrodes were attached to the skin was placed by following the standard protocol. Two electrodes were placed nearly $20 \mathrm{~mm}$ apart in the direction of the muscle fibers.

For UT muscle, the electrode was placed at the middle point of a straight line from the spine of the seventh cervical vertebra to the lateral edge of acromion. ${ }^{13}$ For SCM muscle, the electrode was positioned on skin surface fixed at the midpoint of the SCM, $4 \mathrm{~cm}$ from the mastoid process, along the muscle fibers. ${ }^{14}$ For CES, the electrodes were placed over the muscle belly at approximately the fourth cervical vertebra. ${ }^{15,16}$

The median power frequency (MDF) was used to monitor muscle fatigue. The collected EMG signal of UT, SCM, and CES have been digitally processed by using the Fast Fourier transformation technique of software to analyze the value of the MDF. MDF means the median value from the spectrum of the EMG of the total recording time (10 minutes). The lower the MDF indicates the higher the muscle fatigue while the higher the MDF indicates the lower the muscle fatigue. $^{17}$

\section{2) Visual feedback with using a mobile posture-aware system}

To maintain head angle of subject, we used a mobile posture-aware system to provide visual feedback of head angle. This is the new approach to monitor the posture of smartphone users with built-in sensors. In Android operation system, head-up application 


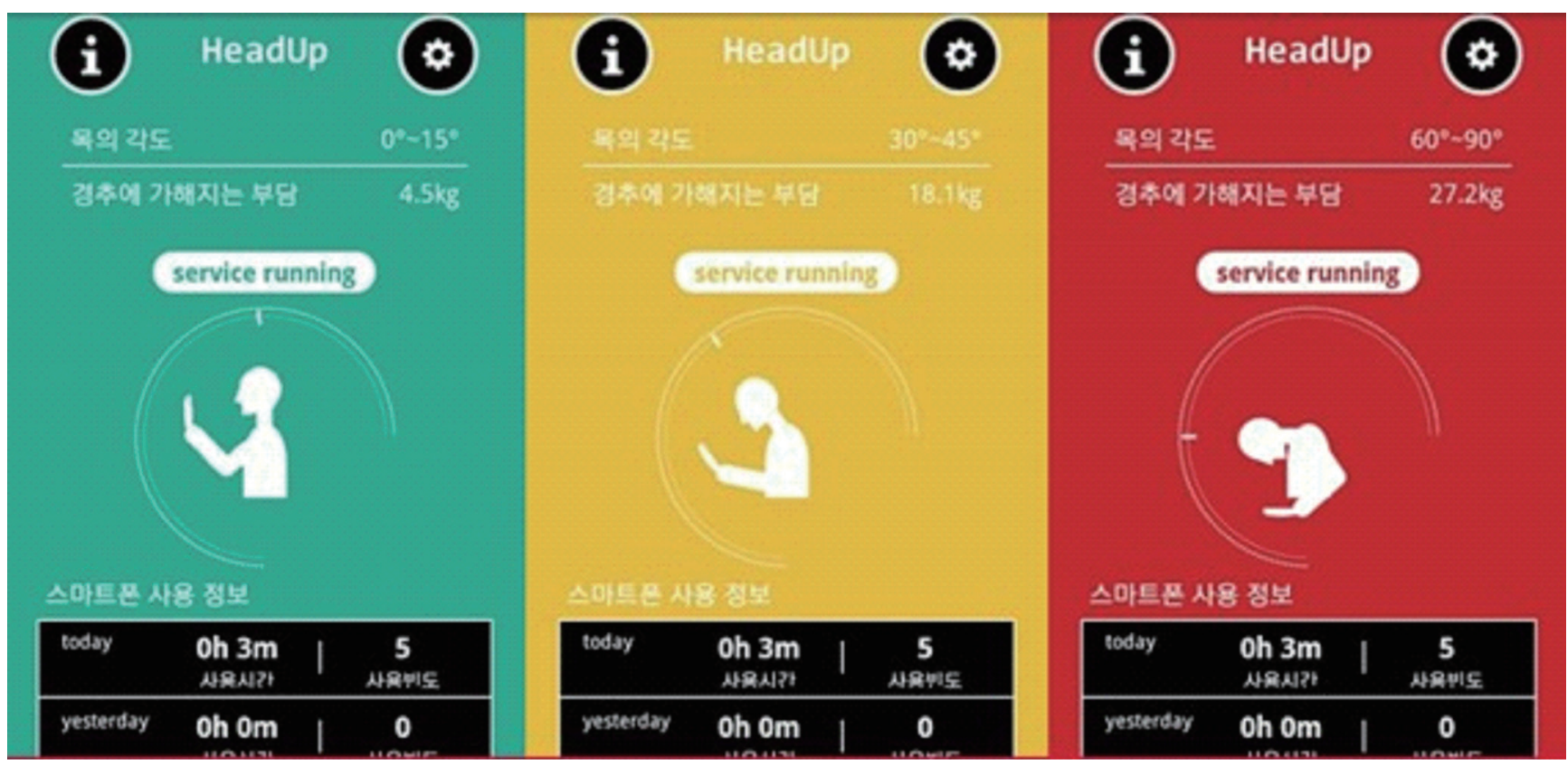

Figure 1. Visual feedback of using a mobile posture-aware system.
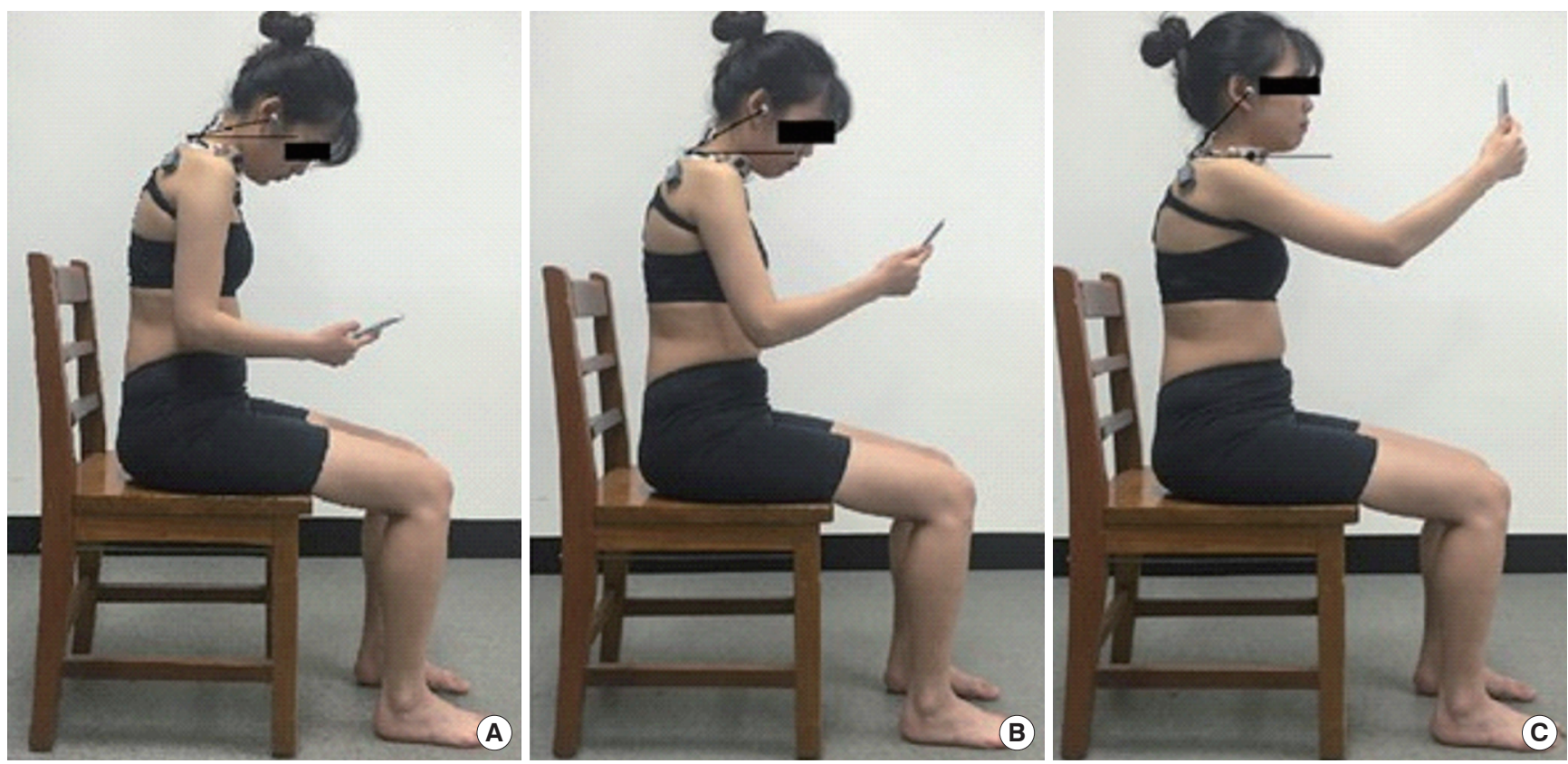

Figure 2. Tested position with visual feedback of using a mobile posture-aware system in (A) $60^{\circ}$, (B) $30^{\circ}$, and (C) $0^{\circ}$

(PAWNSH@P-developer) estimates a value representing head angle of users by evaluating sensor data from a front-faced camera, 3-axis accelerometer, and orientation sensor (Figure 1). ${ }^{3}$

\section{3) Craniocervical angle measurement}

Craniocervical angles were measured using a sagittal-view photograph of the subject in a sitting posture. Two light-reflective markers were positioned over the tragus of the subject's the center of the ear, C7 spinous process, as shown in figure $2 .^{18}$ The craniovertebral angle was recognized at the intersection of a horizontal line passing through the $\mathrm{C} 7$ spinous process and a line connection the center of the tragus of the ear to the $\mathrm{C} 7$ spinous process. To reduce image distortion, primary investigator installed the camera on a tripod to confirm that the camera was perpendicular to the horizontal. Im- 


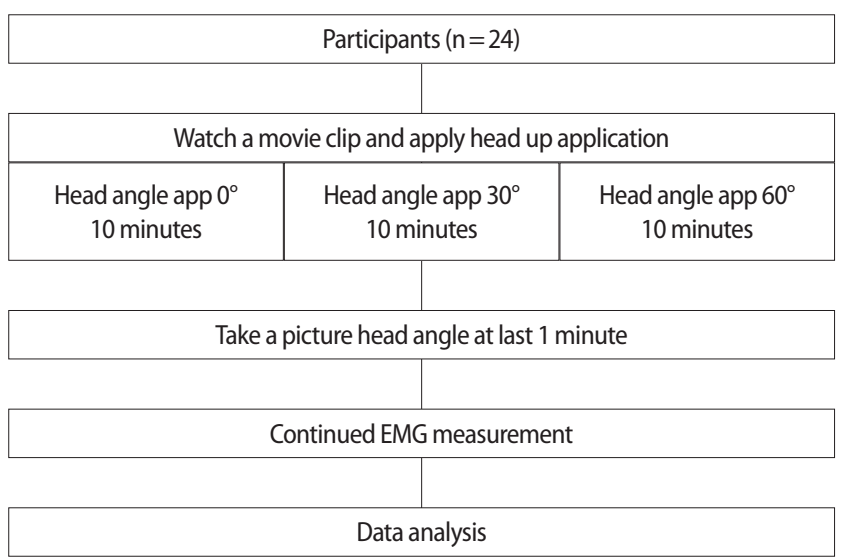

Figure 3. Procedure flow chart.

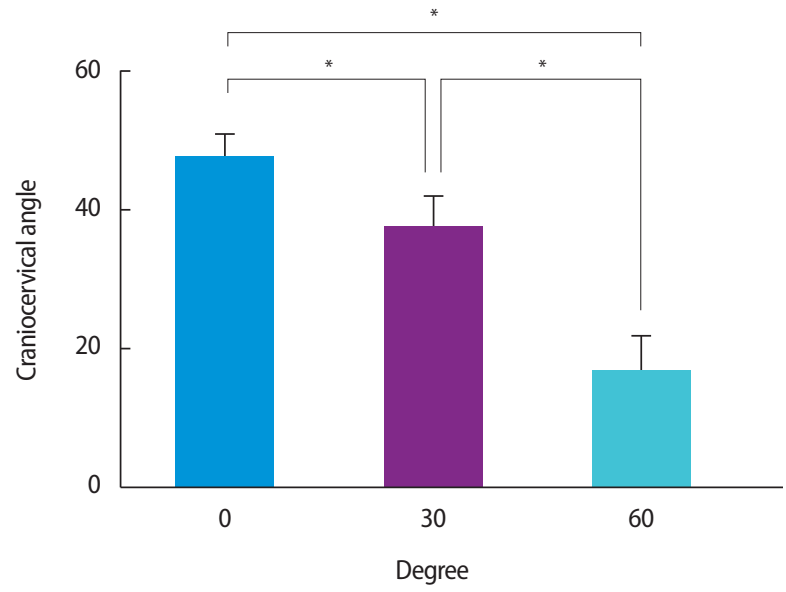

Figure 4. Craniocervical angle after using a smartphone. $\star p=0.001$.

age J software (National Institutes of Health, Bethesda, available at http://www.rsb.info.nih.gov/ij) was used to measure craniocervical angle from the images. CCA measurements showed good intra-rater reliability in previous studies (intra class correlation coefficients of $0.88-0.98)^{18}$

\section{Procedure}

We ask the subject to sit comfortably with maintaining their hip and knee joints at $90^{\circ}$. Then, subject held a smartphone (Galaxy Note 3, Samsung Electronics Co., Ltd., Seoul, Korea) with right hands and started at the screen (Figure 2). The Head-up application was performed to provide the visual feedback of their posture. The subjects started to watch a movie clip for 10 minutes with visual feedback in $0^{\circ}, 30^{\circ}$, and $60^{\circ}$ in a random order generated by an Excel program. A 5 minutes rest was provided between trials to avoid
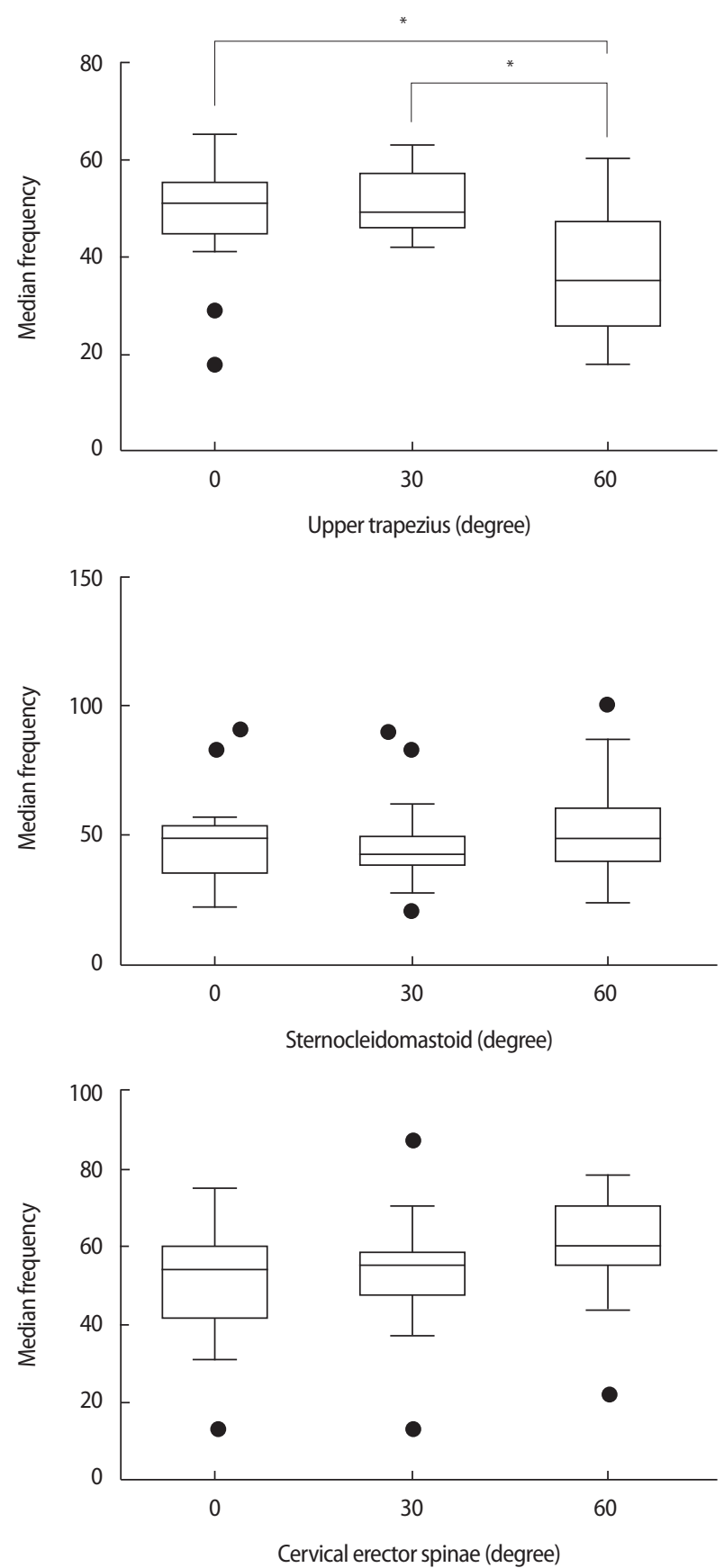

Figure 5. Values of the Median frequency with $0^{\circ}, 30^{\circ}$, and $60^{\circ}$ of visual feedback on neck and shoulder muscles. $\star p=0.001$.

muscle fatigue. ${ }^{19}$ The investigator observed the subjects from the side to ensure that the subjects keep the watching task without other behavior. Craniocervical angle measurement was performed at the last minute of 10 minutes watching a movie clip. The data collection was repeated three times and averaged to decrease measurement errors (Figure 3). 


\section{Statistical analysis}

The Kolmogorov-Smirnov Z-test was executed to examine whether continuous data were a normal distribution. We used a one-way repeated analysis of variance with a significant level of 0.05 to investigate the differences in craniocervical angles and median frequency of neck and shoulder muscles during 10 minutes of watching the smartphone screen with feedback in various angles. Post-hoc analyses were performed during the Bonferroni correction to evaluate the significance of between-exercise pair-wise comparisons. The statistical significance level was set at $\alpha=0.05 / 3=0.0017$. SPSS ver. 22.0 software was performed for all data analysis (SPSS Inc., Chicago, IL, USA).

\section{RESULTS}

All continuous data were normally distributed (KolmogorovSmirnov Z-tests, $\mathrm{p}>0.005$ ). Figure 4 shows the craniocervical angles after using a smartphone in $0^{\circ}, 30^{\circ}$, and $60^{\circ}$ of visual feedback. Pairwise comparisons confirmed that craniocervical angle in $0^{\circ}$ visual feedback (95\% confidence interval: 43.93-50.86) was significantly greater than $30^{\circ}$ (95\% confidence interval: 32.64-42.01, $\left.\mathrm{p}=0.002\right)$ and $60^{\circ}$ (95\% confidence interval: $\left.11.14-21.83, \mathrm{p}=0.001\right)$. In addition, craniocervical angle in $30^{\circ}$ visual feedback (95\% confidence interval: 32.64-42.01) was significantly greater than $60^{\circ}(95 \%$ confidence interval: $11.14-21.83, \mathrm{p}=0.001$ ) as described figure 5 .

To estimate the effect of using a smartphone in $0^{\circ}, 30^{\circ}$, and $60^{\circ}$ of visual feedback on muscle fatigue, MDF have been analyzed. There was significant difference in the MDF of UT muscles among 3 head angles. Bonferroni post-hoc test revealed that MDF of UT muscles in $0^{\circ}$ and $30^{\circ}$ of visual feedback was significantly greater than $60^{\circ}$ $(p=0.001)$. There were no significant differences in the MDF.

\section{DISCUSSION}

The purpose of this study was finding out the effect of visual feedback of head angles $\left(0^{\circ}, 30^{\circ}, 60^{\circ}\right)$ with using a mobile posture-aware system on craniocervical angle and neck and shoulder muscles (UT, SCM, CES) fatigue during 10 minutes watching the smartphone screen. Our result show that there were significant differences among the effect in $0^{\circ}, 30^{\circ}$, and $60^{\circ}$ of visual feedback of head angles on craniocervical angles. Also MDF of UT muscles in $0^{\circ}$ and $30^{\circ}$ of visual feedback was significantly greater than $60^{\circ}$. And, there were no significant differences in the MDF of SCM and CES muscles among 3 head angles.

There were significant differences on craniocervical angle among $0^{\circ}, 30^{\circ}$, and $60^{\circ}$ of visual feedback of head angles. And, the mean value of craniocervical angles at each visual feedback of head angles orderly decreased as $47.40^{\circ}, 37.68^{\circ}$, and $15.78^{\circ}$. Although a previous study proposed to use a new posture monitoring system, clinical trial study has not been researched with human subject. ${ }^{20}$ Therefore, there was no other study to compare. But a research reported that the craniocervical angles significantly increased during video display terminal work when using the proximity sensor with auditory feedback as similar to our study. ${ }^{21}$ Therefore, $0^{\circ}$ visual feedback of head angle with using a mobile posture-aware system would be beneficial to prevent or decrease forward head posture during watching a smartphone.

Our study discovered that MDF of UT muscles in $0^{\circ}$ and $30^{\circ}$ of visual feedback was significantly greater than $60^{\circ}$. This imply that watching a smartphone with $0^{\circ}$ and $30^{\circ}$ of visual feedback for 10 minutes would decrease UT muscle fatigue than $60^{\circ}$ of visual feedback. $0^{\circ}$ and $30^{\circ}$ of visual feedback would present increased shoulder flexion than $60^{\circ}$ of visual feedback. Other previous study presented that higher shoulder flexion would increase risk of UT muscle activity level. ${ }^{22}$ However, Lee et al. ${ }^{23}$ reported that the muscle fatigue of bilateral UT were highest at a cervical flexion angle of $50^{\circ}$ and lowest at an angle of $30^{\circ}$. And, they claimed that the craniocervical flexion angle should be maintained at $30^{\circ}$ rather than $50^{\circ}$ or $0^{\circ} .{ }^{23}$ The other study reported that UT muscle activity was also significantly decreased with increasing of craniocervical angle correction by taping during computer work. ${ }^{8}$ These previous studies indicate that the degree of fatigue of UT is affected by the angle of the neck and the angle of the arm. In conclusion, the posture in $0^{\circ}$ of visual feedback would causes UT muscle fatigue easily than other angles due to increased shoulder flexion angles. In addition, our result showed that $0^{\circ}$ and $30^{\circ}$ of visual feedback showed no statistical differences in MDF of UT. Therefore, we recommend using $30^{\circ}$ of visual feedback in case of caring UT muscle fatigue primarily.

There were no significant differences in the MDF of SCM and CES muscles among 3 head angles. Unlike our expectations, this result imply that the visual feedback of head angles did not effect on SCM and CES muscle fatigue. A previous study reported that there 
are significant differences in SCM and CES muscle activity between $0^{\circ}, 30^{\circ}$, and full of cervical flexion. ${ }^{24}$ The possible reason of the different results would relatively low muscle activity level in smartphone watching task. In study of Lee et al. ${ }^{24}$ the muscle activity level of SCM $\left(0^{\circ}: 3.34 \% \mathrm{MVIC}, 30^{\circ}: 4.56 \% \mathrm{MVIC}\right.$, full of cervical flexion: 5.65\%MVIC) and CES (0: 9.64\%MVIC, 30: 13.22\%MVIC, full of cervical flexion: $13.06 \% \mathrm{MVIC})$ were relatively low intensity. The other study demonstrates that the behavior of the muscle fatigue is strongly influenced by the intensity of the load. And, lower than $20 \%$ MVIC would not enough to generate muscle fatigue in short term. ${ }^{25}$ Consequently, we assumed that 10 minutes watching task among 3 head angles were not sufficient intensity of the load to causes the significant differences in muscle fatigue of SCM and CES.

In recent years, the daily use time of smart phones has increased while people who complain of musculoskeletal pain caused by FHD have increased. Based on our findings, recommending the use of a visual feedback program in proper way when using smartphones will help prevent or reduce FHD.

Our study had some limitation. First of all, we could not control the subject posture and observed a compensation movement such as scapular elevation in the condition of waking with $0^{\circ}$ visual feedback. Second, the design of our study was a cross sectional study. Third, we only investigated the craniocervical angle not the neck flexion angle. Fourth, the evaluation during 10 minutes of smartphone use were insufficient time to cause fatigue in the muscles of SCM and CES. Thus, further study is necessary to investigate the long-term effect of visual feedback of head angle on craniocervical and neck flexion angle.

\section{ACKNOWLEDGEMENTS}

We thank Nu-ri Seol for helping us with our research.

\section{REFERENCES}

1. Arslan A, Ünal AT. Examination of cell phone usage habits and purposes of education faculty students. IJHS. 2013;10(1):182-201.

2. Park JH, Kim JH, Kim JG et al. The effects of heavy smartphone use on the cervical angle, pain threshold of neck muscles and depression. Adv Sci Technol Lett. 2015;91:12-7.

3. Lee HS, Choi YS, Lee SJ et al. A new posture monitoring system for preventing physical illness of smartphone users. IEEE. 2013:713-16.
4. Fernández-de-las-Peñas C, Pérez-de-Heredia M, Molero-Sánchez A et al. Performance of the craniocervical flexion test, forward head posture, and headache clinical parameters in patients with chronic tension-type headache: a pilot study. J Orthop Sports Phys Ther. 2007;37(2):33-9.

5. Raine S, Twomey LT. Head and shoulder posture variations in 160 asymptomatic women and men. Arch Phys Med Rehabil. 1997;78(11):121523.

6. Thomas K, Cureton JR. Bodily posture as an indicator of fitness. Res Q Exerc Sport. 1941;12:348-67.

7. Kim MH, Yi CH, Kwon OY et al. Changes in neck muscle electromyography and forward head posture of children when carrying schoolbags. Ergonomics. 2008;51(6):890-901.

8. Yoo W. Effect of the neck retraction taping (NRT) on forward head posture and the upper trapezius muscle during computer work. J Phys Ther Sci. 2013;25(5):581-2.

9. Kim EJ, Kim JW, Park BR. Effects of sling exercise program on muscle activity and cervical spine curvature of forward head posture. Jour of KoCon.a. 2011;11(11):213-20.

10. Harman K, Hubley-Kozey CL, Butler H. Effectiveness of an exercise program to improve forward head posture in normal adults: a randomized, controlled 10-week trial. J Man Manip Ther. 2005;13(3):163-76.

11. Morningstar MW, Strauchman MN, Weeks DA. Spinal manipulation and anterior head weighting for the correction of forward head posture and cervical hypolordosis: a pilot study. Chiropr Med. 2003;2(2):51-4.

12. Quek J, Pua YH, Clark RA et al. Effects of thoracic kyphosis and forward head posture on cervical range of motion in older adults. Man Ther. 2013;18(1):65-71.

13. Jensen C, Vasseljen O, Westgaard RH. The influence of electrode position on bipolar surface electromyogram recordings of the upper trapezius muscle. Eur J Appl Physiol Occup Physiol. 1993;67(3):266-73.

14. Tarmin SB, Akir NF, Guan NY et al. The effect of using dual screen computer panel to the neck-shoulder muscle activity among group of students. Iran J Public Health. 2016;45(1):61-7.

15. Cram JR, Kasman GS, Holtz J. Introduction to surface electromyography. Gaithersburg, Aspen Publishers, 1998.

16. Farina D, Madeleine P, Graven-Nielsen T et al. Standardising surface electromyogram recordings for assessment of activity and fatigue in the human upper trapezius muscle. Eur J Appl Physiol. 2002;86:469-78.

17. Murata A, Uetake A, Matsumoto S et al. Evaluation of shoulder muscular fatigue induced during mouse operation in a VDT task. IEICE. 2005; 88(2):223-9.

18. Kuo YL, Tully EA, Galea MP. Video analysis of sagittal spinal posture in healthy young and older adults. J Manipulative Physiol Ther. 2009;32(3): 210-5.

19. Park S, Yoo W. Effect of EMG-based feedback on posture correction during computer operation. J Occup Health. 2012;54(4):271-7.

20. Lee HS, Choi YS, Lee SJ et al. Smart pose: mobile posture-aware system for lowering physical health risk of smartphone users. CHI'13 Extended Abstracts on Human Factors in Computing Systems. 2013:2257-66.

21. Yoo W, Yi C, Kim M. Effects of a proximity-sensing feedback chair on head, shoulder, and trunk postures when working at a visual display terminal. J Occup Rehabil. 2006;16(4):631-7.

22. Kong YK. The effects of co-ordinating postures with shoulder and elbow flexion angles on maximum grip strength and upper-limb muscle activity 
in standing and sitting postures. Int J Occup Saf Ergon. 2014;20(4):595606.

23. Lee SY, Lee DH, Park JS. Effect of the cervical flexion angle during smart phone use on muscle fatigue of the cervical erector spinae and upper trapezius. J Phys Ther Sci. 2015;(27):1847-9.

24. Lee TH, Lee JH, Lee YS et al. Changes in the activity of the muscles sur- rounding the neck according to the angles of movement of the neck in adults in their 20s. J Phys Ther Sci. 2015;27(3):973.

25. Silva CR da, Silva D de O, Ferrari D et al. Exploratory study of electromyographic behavior of the vastus medialis and vastus lateralis at neuromuscular fatigue onset. Motriz Rev Ed Fis. 2014;20(2):213-20. 